\title{
Top-down and bottom-up sequential modulations of congruency effects
}

\author{
WIM NOTEBAERT, WIM GEVERS, FREDERICK VERBRUGGEN, and BAPTIST LIEFOOGHE \\ Ghent University, Ghent, Belgium
}

\begin{abstract}
Several studies have demonstrated reduced congruency effects after incongruent trials. The conflict monitoring hypothesis (Botvinick, Braver, Barch, Carter, \& Cohen, 2001) assumes that this sequential modulation is based on top-down cognitive control and suggests that more control is engaged after the detection of conflict. An alternative account is based on repetition effects of stimulus and response features and can be considered bottom up. This study investigates both modulatory sources. In a Stroop task with two response-stimulus intervals (RSIs), we demonstrate that top-down modulation does not occur with a very short RSI, suggesting that it takes some time before the system can be reconfigured. Bottom-up modulation is observed for both RSIs. This finding demonstrates that two different sources simultaneously reduce congruency effects after incongruent trials.
\end{abstract}

Congruency effects, such as the Simon, Stroop, and flanker effects, are generally described as failures in selective attention, since participants typically fail to ignore irrelevant information. In a Simon task, a spatial response (left or right key) is assigned to the color or shape of a stimulus, and stimuli are randomly presented to the left or right of a fixation cross. Although the stimulus location is irrelevant for the task, reaction times (RTs) are faster when the stimulus and the response locations correspond (Simon, 1990). In a Stroop task, responses are assigned to the color of a word, and the word meaning itself is irrelevant. Results typically show faster RTs when the color and the irrelevant word meaning are congruent (when the word GREEN is in green) than when they are incongruent (when GREEN is in red; for an overview, see MacLeod, 1991). In a flanker task, the flankers presented next to the target are irrelevant, but RTs are faster when target and flankers elicit the same response (Eriksen \& Eriksen, 1974). In an arrow flanker task, for instance, participants have to respond to a central arrow and ignore the flankers, and the results usually show faster RTs on congruent $(>>>)$ than on incongruent $(><>)$ trials.

Congruency effects are usually explained in terms of dual-route models (De Jong, Liang, \& Lauber, 1994; Kornblum, Hasbroucq, \& Osman, 1990). The relevant information is processed through a controlled route that is relatively slow because it arbitrarily assigns a response to a particular stimulus feature (green corresponds to a left response, for instance). Another, fast route (the automatic

W.G. is supported by Grant D.0353.01 from the Flemish Fund for Scientific Research. F.V. is a fellow (Grant 011D06102) of the Special Research Fund at Ghent University. We thank Wilfried Kunde and two anonymous reviewers for their appreciation and useful comments. Correspondence relating to this article may be sent to W. Notebaert, Department of Experimental Psychology, Ghent University, Henri Dunantlaan 2, 9000 Ghent, Belgium (e-mail: wim.notebaert@ugent.be). priming route) processes the irrelevant information and preactivates (primes) a particular response. When the required response is the response that was primed, RTs will be faster than when another response is required.

In serial reaction time tasks, it has been demonstrated that congruency effects, measured as the difference between congruent and incongruent trials, depend on the congruency of the preceding trial. When the previous trial (trial $n-1$ ) was incongruent (GREEN in red), the congruency effect will be weaker than for trials in which trial $n-1$ was congruent (Kerns et al., 2004). Similar effects have been observed for the Simon effect (Notebaert, Soetens, \& Melis, 2001; Stürmer, Leuthold, Soetens, Schröter, \& Sommer, 2002), the flanker effect (Gratton, Coles, \& Donchin, 1992), and prime-target correspondence effects (Kunde, 2003).

Botvinick, Braver, Barch, Carter, and Cohen (2001) proposed a conflict monitoring hypothesis in order to integrate these behavioral effects with the results of brain imaging studies. Several brain imaging studies have demonstrated higher activation in the anterior cingulate cortex on incongruent than on congruent trials (Kerns et al., 2004; Pardo, Pardo, Janer, \& Raichle, 1990). This activation was interpreted as the detection of conflict (Botvinick, Nystrom, Fissell, Carter, \& Cohen, 1999). The conflict monitoring hypothesis further states that whenever conflict is detected, top-down reconfiguration takes place and more control is allocated to the particular task. The purpose of this reconfiguration is to eliminate, or at least reduce, the influence of the irrelevant information.

Several authors have claimed that there is an alternative explanation for this sequence effect (Hommel, Proctor, \& Vu, 2004; Mayr, Awh, \& Laurey, 2003; Notebaert et al., 2001). The idea behind this alternative hypothesis is that certain transitions from trial $n-1$ to trial $n$ lead to faster RTs than other transitions do. RTs are usually fast when both stimulus features (color and word in the Stroop task) 
are repeated, but they are also relatively fast when both features change. Relatively slow RTs are observed when one of the features is repeated and another changes. In general, this is explained in terms of a binding process (Hommel, 1998; Hommel et al., 2004; Notebaert \& Soetens, 2003; Notebaert et al., 2001). On any given trial, the stimulus and response features are temporarily associated with each other. When the next trial violates this association, RTs are slow. This is the case when one of the features is repeated and another changes. These repetition and binding effects artificially influence the size of congruency effects. As an example, consider a Stroop task with two colors/responses and two irrelevant words. First, take the case in which trial $n-1$ was congruent (GREEN in green). If the present trial is congruent, a relatively fast response is expected because the trial is either a complete repetition (GREEN in green) or a complete alternation (RED in red). If the present trial is incongruent (GREEN in red), a relatively slow response is expected because either the stimulus word (GREEN in red) or the response (RED in green) is repeated, but never both. Therefore, after congruent trials, a relatively large congruency effect can be expected on the basis of these repetition effects. Now consider the case in which trial $n-1$ was incongruent (GREEN in red). If the present trial is congruent, it will be relatively slow because either the stimulus word (GREEN in green) or the response (RED in red) is repeated, but not both. If the present trial is incongruent, this is because a complete repetition (GREEN in red) or a complete alternation (RED in green) occurred, resulting in fast RTs. As a result, after incongruent trials, a reduced congruency effect can be expected. In general, smaller congruency effects after incongruent than after congruent trials can, at least to some extent, be attributed to bottom-up repetition effects.

Recently, this hypothesis received some empirical support (Hommel et al., 2004; Mayr et al., 2003). In a flanker task with arrows, Mayr et al. demonstrated that, when target (response) repetitions were excluded from the data analysis, there was no adaptation of the flanker effect after incongruent trials. This result suggests that the adaptation of congruency effects can indeed be an artifact of repetition effects. A couple of recent studies, on the other hand, controlled for these repetition effects and still observed an interaction between the congruency of the previous and the congruency of the current trial. Kerns et al. (2004) used a Stroop task with three colors and three words, making it possible to focus on complete alternations only and to exclude the confound of repetition effects. For complete alternations, Kerns et al. observed a reduced Stroop effect after incongruent trials. In addition, Wühr (2005) held (partial) repetitions constant and still observed a sequential modulation of the Simon effect. In general, it seems that when one controls for repetition effects, top-down conflict adaptation still occurs.

In this study, we used a Stroop task with three colors and three words. In order to study top-down conflict adaptation, we focused on complete alternations (Kerns et al., 2004). We also examined the remaining trials, in which the word, the color, or both features repeated. For these trials, we expected a strong impact of bottom-up repetition effects. We studied top-down adaptation and bottomup effects with two response-stimulus intervals (RSIs). We expected that the length of the interval between the response on the previous trial and the onset of the new stimulus would mainly affect top-down adaptation. Since we used two very short RSIs (50 and $200 \mathrm{msec}$ ), we expected no difference in terms of repetition effects. For the top-down adaptation, on the other hand, we expected that some time would be needed between the detection of the conflict and the reconfiguration of the processing system. It was assumed that conflict detection occurs only slightly before response execution (Burle, Allain, Vidal, \& Hasbroucq, 2005; Van Veen \& Carter, 2002), and it has been demonstrated in a variety of tasks that top-down attentional control requires a fair amount of time (Posner, 1980). In general, it seems that endogenous (top-down) control cannot alter the focus of attention when less than 100-150 msec elapse between the trigger and the stimulus (see also Müller \& Rabbitt, 1989). We used two RSIs (50 and $200 \mathrm{msec}$ ) and expected that top-down reconfiguration could not take place with an interval of approximately $50 \mathrm{msec}$ between detection of a conflict and the onset of a new stimulus. With a 200-msec interval, we expected there would be enough time to reconfigure the system after a conflict situation. Thus, for complete alternations, only in the 200-msec condition did we expect a stronger Stroop effect after congruent than after incongruent trials.

In sum, to investigate the temporal aspects of top-down conflict monitoring, we analyzed complete alternations. We expected adaptation of the Stroop effect only after incongruent trials in the long-RSI condition. For the remaining trials, in which at least one of the features was repeated, we expected a large influence of bottom-up repetition effects, and thus a reduced congruency effect after incongruent trials in both RSI conditions.

\section{METHOD}

\section{Participants}

Thirty-four participants with normal or corrected-to-normal vision were tested at Ghent University. Seventeen (ages 18-35; 6 males and 11 females) took part in the short-RSI condition $(50 \mathrm{msec})$ and 17 (ages 18-35; 4 males and 13 females) in the long-RSI condition (200 msec).

\section{Materials and Procedure}

Participants were tested on Pentium computers running E-Prime software(Psychology SoftwareTools,Inc.; www.pstnet.com/products/ e-prime/). The stimuli consisted of the words GROEN (green), GEEL (yellow), and ROOD (red) presented in Arial capitals in the colors green, yellow, and red. The words were approximately $2 \mathrm{~cm}$ wide and $0.5 \mathrm{~cm}$ high. Participants had to react as fast as possible to the stimulus color with the index, middle, or ring finger of their dominant hand on the "c," "v," or "b" keys of a standard keyboard. Stimuli were presented in the center of a computer display approximately $50 \mathrm{~cm}$ from the participants and remained on the screen until a response key was pressed. This keypress started the RSI, which was set at $50 \mathrm{msec}$ or $200 \mathrm{msec}$ (between subjects). During the RSI, a gray fixation cross was presented. The nine stimulus combinations were presented in random order, which resulted in 33\% congruent trials and $66 \%$ incongruent trials. On $33 \%$ of the trials, the irrelevant word 
was repeated, just as on $33 \%$ of the trials the relevant color (response repetitions) was repeated. Participants ran through a practice block of 90 trials, followed by 8 blocks of 90 trials.

\section{RESULTS}

We excluded every first trial from a block, errors, and trials following an error. This procedure excluded 5.8\% of the trials in the 50-msec condition and 5.3\% in the 200-msec condition. RTs shorter than $100 \mathrm{msec}$ and longer than 3,000 msec were also excluded. This cutoff procedure excluded less than $1 \%$ of the remaining trials. An ANOVA with two within-subjects factors (congruency on trial $n-1$ and congruency on trial $n$ ) and one betweensubjects factor (RSI) was conducted on the mean RTs.

\section{Complete Alternations Trials}

In order to study the temporal aspects of top-down conflict adaptation, we analyzed trials in which both the color and the word changed from the previous trial. The results are shown in Figure 1, which reveals different patterns across the two RSI conditions. In the 50 -msec condition (left panel), the Stroop effect is similar after congruent and incongruent trials. In the 200-msec condition (right panel), the Stroop effect is larger after congruent than after incongruent trials.

There was no overall RT difference between the RSI conditions $[F(1,32)=1.52, p=.23]$. There was an overall Stroop effect $[F(1,32)=88.82, p<.001]$, but more importantly, the Stroop effect interacted with the congruency of trial $n-1[F(1,32)=9.30, p<.01]$. There was also a significant three-way interaction $[F(1,32)=4.64, p<$
$.05]$ : In the short-RSI condition, there was no interaction between previous and current congruency $[F(1,32)<1$, n.s.], but in the 200-msec condition an interaction was observed $[F(1,32)=13.54, p<.001]$.

\section{Complete and Partial Repetition Trials}

The remaining trials consisted of different types of transitions in terms of repetitions. After congruent trials, a congruent trial could only be a complete repetition and an incongruent trial could only be a partial repetition. Consequently, we expected a large difference between congruent and incongruent trials. After incongruent trials, a reduced Stroop effect was expected, since congruent trials are slow partial repetitions, and incongruent trials are fast complete repetitions. Figure 2 shows that there was no difference between the two RSI conditions. After a congruent trial, there was a large Stroop effect, and after an incongruent trial there was no Stroop effect.

There was no overall RT difference between the two RSI conditions $[F(1,32)=1.70, p=.20]$, and there was an overall Stroop effect $[F(1,32)=108.80, p<.001]$. The interaction between the congruency of $n-1$ and the congruency of $n$ was significant $[F(1,32)=124.22, p<.001]$, but the three-way interaction was not $[F(1,32)<1$, n.s. $]$.

\section{DISCUSSION}

The word and color alternations of a Stroop task with an RSI of $200 \mathrm{msec}$ revealed a typical conflict monitoring pattern, in which the Stroop effect was smaller after an incongruent trial than after a congruent trial. However, with an RSI of $50 \mathrm{msec}$, a similar Stroop effect was observed
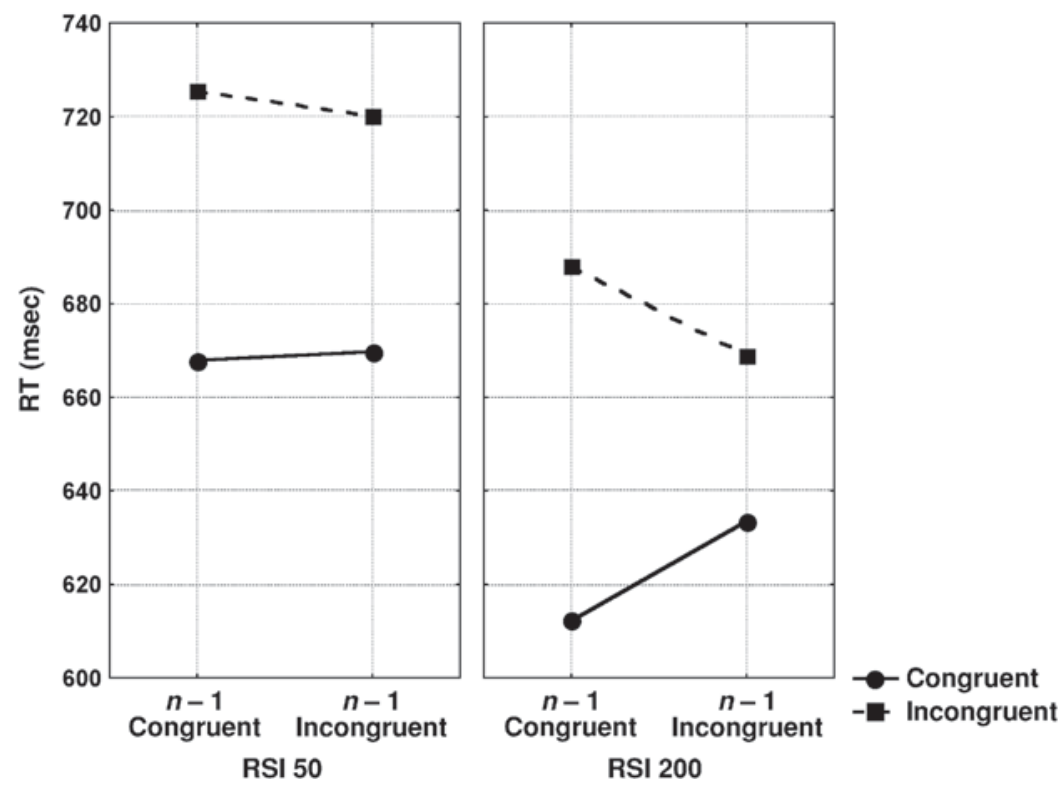

Figure 1. Reaction times (RTs) for congruent and incongruent trials as a function of the congruency of the previous trial. Only complete alternations of word and color are included. The left panel shows RTs for the 50-msec condition, the right panel for the 200-msec condition. 

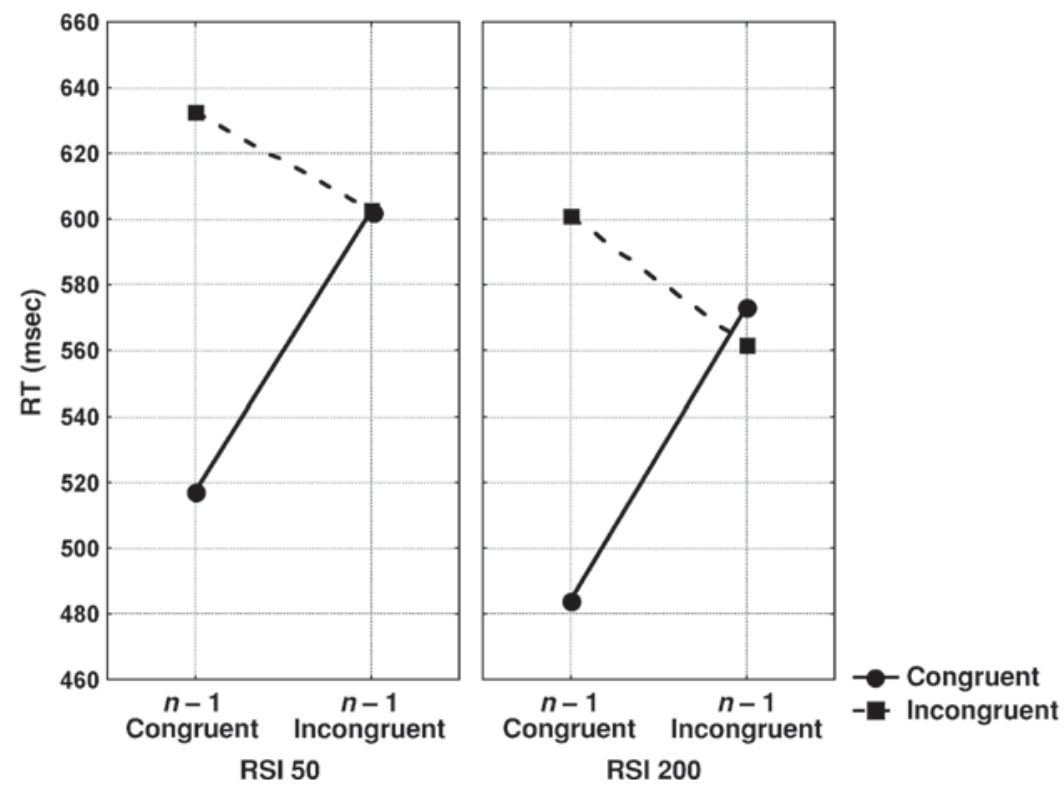

Figure 2. Reaction times (RTs) for congruent and incongruent trials as a function of the congruency of the previous trial. Only trials in which at least one of the two features (color or word) was repeated are included. The left panel shows RTs for the 50-msec condition, the right panel for the 200-msec condition.

after congruent and incongruent trials. When we analyzed trials in which at least one of the features was repeated, a different pattern emerged: In both RSI conditions, the Stroop effect completely disappeared after incongruent trials. The present data set has important theoretical implications.

First, conflict monitoring was observed for complete alternations. This observation replicates the results of Kerns et al. (2004). The emerging picture is that an interaction between previous and current congruency is observed, even when the design is carefully balanced against repetition effects (Wühr, 2005) or when repetitions are excluded altogether (Kerns et al., 2004). According to the conflict monitoring hypothesis (Botvinick et al., 2001), the conflict on trial $n-1$ is detected by the anterior cingulate cortex, and more control is allocated. This extra control reduces the influence of the irrelevant information, and consequently decreases the congruency effect. How this top-down control is achieved remains unclear, although some suggestions have been made. For the adaptation in the Simon effect, Stürmer et al. (2002) proposed that sensorimotor pathways are modulated. In particular, they assumed that the output of the automatic priming route is not transmitted to the motor execution system after incongruent trials. They based this hypothesis on ERP observations that incorrect response activation occurred in the posterior parietal cortex but not in the motor cortex following incongruent trials. Wühr and Ansorge (2005) added that the modulation works in both directions: After congruent trials, the conductivity to motor areas is enhanced, but after incongruent trials, the conductivity is reduced. More research is needed in order to understand how this con- ductivity is regulated, but on the basis of our results, we know that this regulation needs some time, since conflict monitoring is not observed with an RSI of $50 \mathrm{msec}$. This is in line with the temporal aspects of top-down attentional control (see, e.g., Müller \& Rabbitt, 1989). In cuing paradigms, researchers have observed on several occasions that endogenous cues (requiring top-down control) cannot alter the focus of attention when the cue-target interval is too short (less than 100-150 msec). Investigating the other side of the time line, Wühr and Ansorge recently showed that sequential modulations were more pronounced with a stimulus onset asynchrony (SOA) of $1.5 \mathrm{sec}$ than with an SOA of $6 \mathrm{sec}$. When there is too much time between the detection of a conflict and the onset of the new stimulus, the effect of the reconfiguration has decayed.

The reason why Mayr et al. (2003) did not observe topdown conflict monitoring in the flanker task (after removing target repetitions) could be that two consecutive incongruent trials were always negative-priming transitions in their task $(><>$ followed by $<><$; see also Egner $\&$ Hirsch, 2005). Negative-priming transitions are usually slower than other transitions because the irrelevant tobe-ignored information of the previous trial becomes the relevant information on the following trial (for an overview, see Tipper, 2001). This could artificially increase the flanker effect after incongruent trials and hide a conflict monitoring pattern.

When we focused on trials in which at least one of the features was repeated, we observed no Stroop effect after incongruent trials. Both RSIs elicited identical patterns for this subset of the data, and this pattern is best described in terms of repetition effects. After a congruent trial, a 
large Stroop effect is observed, because congruent trials represent fast complete repetitions and incongruent trials represent slow partial repetitions. After incongruent trials, a reduced Stroop effect is observed, because congruent trials are now slow partial repetitions and incongruent trials are fast complete repetitions.

For partial and complete repetitions, there is no reason not to expect top-down adjustment. This means that in the 200-msec condition, the interaction observed in Figure 2 is the additive effect of top-down and bottom-up modulations. But this should mean that the interaction in the 50 -msec condition should be weaker, and this is not the case. It is important to note that in the 50 -msec condition, the congruency effect completely disappeared after incongruent trials. In order to observe a stronger interaction in the 200-msec condition (on the basis of top-down control), a stronger Stroop effect after congruent trials or a reverse Stroop effect after incongruent trials would have to be observed. From a theoretical point of view, it can be debated whether such results are possible. Besides this theoretical argument, there are several hypotheses that could explain why the interaction between previous and current congruency is not stronger in the 200-msec condition. First, it is possible that bottom-up effects are stronger in the $50-\mathrm{msec}$ condition, but this theory is not supported by a comparison of the response repetition effects in both conditions. In both conditions, response repetitions were faster than response alternations (in the 50-msec condition, 542 vs. $702 \mathrm{msec}$, respectively, and in the 200 -msec condition, 514 vs. $656 \mathrm{msec}$, respectively), and the response repetition effect was not different for the two conditions $[F(1,32)<1$, n.s.], suggesting that bottom-up effects were comparable in both conditions. Another possibility is that the bottom-up and top-down effects interact. This idea comes from studies in which an interaction between congruency effects and repetition effects was observed. In general, congruency effects seem to be reduced by stimulus and response repetition effects. Notebaert et al. (2001) and Notebaert and Soetens (2006) have demonstrated that the Simon, Stroop, and flanker effects are reduced when the irrelevant information is repeated. Others (Bertelson, 1961; Soetens, Boer, \& Hueting, 1985) have demonstrated that congruency effects are smaller for response repetitions. These observations suggest that for (partial) repetition trials, only weak congruency effects are observed. Consequently, the congruency effects observed in Figure 2 are perhaps not really caused by a true difference between congruent and incongruent trials, but rather by bottom-up repetition effects. When there is in fact no true congruency effect for these trials, it is not surprising to observe no top-down adjustment of the congruency effect.

In conclusion, we have shown that two modulatory sources result in smaller congruency effects after incongruent trials. One process reflects top-down reconfiguration and is best described in terms of conflict monitoring. When stimuli appear in extremely fast succession, however, there is no time to make such top-down adjust- ments. Another source that creates a similar interaction is a bottom-up process, caused by stimulus and response repetitions. We observed this bottom-up effect in both RSI conditions.

\section{REFERENCES}

BerTelson, P. (1961). S-R relationships and reaction times to new versus repeated signals in a serial task. Journal of Experimental Psychology, 65, 478-484.

Botvinick, M. M., Braver, T. S., Barch, D. M., Carter, C. S., \& Cohen, J. D. (2001). Conflict monitoring and cognitive control. Psychological Review, 108, 624-652.

Botvinick, M. [M.], Nystrom, L. E., Fissell, K., Carter, C. S., \& Cohen, J. D. (1999). Conflict monitoring versus selection-for-action in anterior cingulate cortex. Nature, 402, 179-181.

Burle, B., Allain, S., Vidal, F., \& HasbroucQ, T. (2005). Sequential compatibility effects and cognitive control: Does conflict really matter? Journal of Experimental Psychology: Human Perception \& Performance, 31, 831-837.

De Jong, R., Liang, C.-C., \& Lauber, E. (1994). Conditional and unconditional automaticity: A dual-process model of effects of spatial stimulus-response correspondence. Journal of Experimental Psychology: Human Perception \& Performance, 20, 731-750.

Egner, T., \& Hirsch, J. (2005). The neural correlates and functional integration of cognitive control in a Stroop task. NeuroImage, 24, 539-547.

Eriksen, B. A., \& Eriksen, C. W. (1974). Effects of noise letters upon the identification of a target letter in a nonsearch task. Perception \& Psychophysics, 16, 143-149.

Gratton, G., Coles, M. G. H., \& Donchin, E. (1992). Optimizing the use of information: Strategic control of activation of responses. Journal of Experimental Psychology: General, 121, 480-506.

Hommel, B. (1998). Event files: Evidence for automatic integration of stimulus-response episodes. Visual Cognition, 5, 183-216.

Hommel, B., Proctor, R. W., \& Vu, K.-P. L. (2004). A feature-integration account of sequential effects in the Simon task. Psychological Research, 68, 1-17.

Kerns, J. G., Cohen, J. D., MacDonald, A. W., III, Cho, R. Y., Stenger, V. A., \& CARTer, C. S. (2004). Anterior cingulate conflict monitoring and adjustments in control. Science, 303, 1023-1026.

Kornblum, S., Hasbroucq, T., \& Osman, A. (1990). Dimensional overlap: Cognitive basis for stimulus-response compatibility. A model and taxonomy. Psychological Review, 97, 253-270.

Kunde, W. (2003). Sequential modulations of stimulus-response correspondence effects depend on awareness of response conflict. Psychonomic Bulletin \& Review, 10, 198-205.

MACLEOD, C. M. (1991). Half a century of research on the Stroop effect: An integrative review. Psychological Bulletin, 109, 163-203.

Mayr, U., Awh, E., \& LAUrey, P. (2003). Conflict adaptation effects in the absence of executive control. Nature Neuroscience, 6, 450-452.

Müller, H. J., \& RabBitT, P. M. A. (1989). Reflexive and voluntary orienting of visual attention: Time course of activation and resistance to interruption. Journal of Experimental Psychology: Human Perception \& Performance, 15, 315-330.

Notebaert, W., \& Soetens, E. (2003). The influence of irrelevant stimulus changes on stimulus and response repetition effects. Acta Psychologica, 112, 143-156.

Notebaert, W., \& Soetens, E. (2006). Sustained suppression in congruency tasks. Quarterly Journal of Experimental Psychology, 59, 178-189.

Notebaert, W., Soetens, E., \& Melis, A. (2001). Sequential analysis of a Simon task: Evidence for an attention-shift account. Psychological Research, 65, 170-184.

Pardo, J. V., Pardo, P. J., Janer, K. W., \& Raichle, M. E. (1990). The anterior cingulate cortex mediates processing selection in the Stroop attentional conflict paradigm. Proceedings of the National Academy of Sciences, 87, 256-259.

PosNer, M. I. (1980). Orienting of attention. Quarterly Journal of Experimental Psychology, 32, 3-25. 
Simon, J. R. (1990). The effects of an irrelevant directional cue on human information processing. In R. W. Proctor \& T. G. Reeve (Eds.), Stimulus-response compatibility: An integrated perspective (pp. 3186). Amsterdam: Elsevier, North-Holland.

Soetens, E., Boer, L. C., \& Hueting, J. E. (1985). Expectancy or automatic facilitation? Separating sequential effects in two-choice reaction time. Journal of Experimental Psychology: Human Perception \& Performance, 11, 598-616.

Stürmer, B., Leuthold, H., Soetens, E., Schröter, H., \& Sommer, W. (2002). Control over location-based response activation in the Simon task: Behavioral and electrophysiological evidence. Journal of Experimental Psychology: Human Perception \& Performance, 28, 13451363.

TIPPER, S. P. (2001). Does negative priming reflect inhibitory mecha- nisms? A review and integration of conflicting views. Quarterly Journal of Experimental Psychology, 54B, 321-343.

Van Veen, V., \& Carter, C. S. (2002). The anterior cingulate as a conflict monitor: fMRI and ERP studies. Physiology \& Behavior, 77, 477-482.

WüHR, P. (2005). Evidence for gating of direct response activation in the Simon task. Psychonomic Bulletin \& Review, 12, 282-288.

WÜHR, P., \& ANSORGE, U. (2005). Exploring trial-by-trial modulations of the Simon effect. Quarterly Journal of Experimental Psychology, 58A, 705-731.

(Manuscript received January 7, 2005; revision accepted for publication May 18, 2005.) 